\title{
Interference effects in the UV(VUV)-excited luminescence spectroscopy of thin dielectric films
}

\section{Evgeny Buntov and Anatoly Zatsepin}

J. Synchrotron Rad. (2013). 20, 509-514

\section{IU IUCr Journals \\ CRYSTALLOGRAPHY JOURNALS ONLINE}

Copyright (C) International Union of Crystallography

Author(s) of this paper may load this reprint on their own web site or institutional repository provided that this cover page is retained. Republication of this article or its storage in electronic databases other than as specified above is not permitted without prior permission in writing from the IUCr.

For further information see http://journals.iucr.org/services/authorrights.html 
Journal of

Synchrotron

Radiation

ISSN 0909-0495

Received 31 October 2012

Accepted 28 January 2013

\section{Interference effects in the UV(VUV)-excited luminescence spectroscopy of thin dielectric films}

\author{
Evgeny Buntov* and Anatoly Zatsepin
}

Institute of Physics and Technology, Ural Federal University, Mira St 19, Ekaterinburg 620002, Russian Federation. E-mail: e.a.buntov@ustu.ru

\begin{abstract}
The problem of exciting UV and VUV light interference affecting experimental photoluminescence excitation spectra is analysed for the case of thin transparent films containing arbitrarily distributed emission centres. A numerical technique and supplied software aimed at modelling the phenomenon and correcting the distorted spectra are proposed. Successful restoration results of the experimental synchrotron data for ion-implanted silica films show that the suggested method has high potential.
\end{abstract}

C 2013 International Union of Crystallography

Printed in Singapore - all rights reserved

\section{Introduction}

Prospective photonic devices require optical components (light sources, switches, mirrors, etc.) to be incorporated into low-dimensional matrices whose size is comparable with or less than the light wavelength. In the case of a tiny light source placed within thin films, linear or planar waveguides, one cannot neglect the interference effects which may affect the performance of optical circuits. Within the model of the electric dipoles spread throughout a thin dielectric layer, emission interference effects, including multiple-beam and wide-angle effects, have been addressed both theoretically and experimentally (Holm et al., 1982). Nevertheless, the problem of the influence of excitation radiation interference was not examined thoroughly.

The question of incident light interference is especially important for a particular type of light source, i.e. photoluminescent quantum dots, non-uniformly distributed inside plane-parallel thin films (Zatsepin et al., 2012a). Being valid even for laser excitation, this phenomenon deserves special attention when a selective synchrotron radiation excitation with a variable wavelength is applied. This may cause periodic oscillations of the photoluminescence excitation (PLE) spectra, thus preventing adequate analysis of these spectra, and therefore hinders the revealing of the nature of the luminescent centres.

During photoluminescent studies of $\mathrm{SiO}_{2}$ films implanted with $\mathrm{Si}$, $\mathrm{C}, \mathrm{S}$ and $\mathrm{Sn}$ ions carried out at DESY (beamline I, SUPERLUMI station), unusual excitation spectra containing a series of regularly spaced bands (oscillations) of similar widths were observed (Zatsepin et al., 2009, 2012a,b; Buntov et al., 2011). It was hypothesized that the origin of these bands was related to interference effects that may occur for the incident electromagnetic waves in films with thicknesses close to the wavelength. The influence of the interference effects on the photoluminescence spectra of thin silica films with silicon nanoclusters was described by Smirani et al. (2005), where IMD software (Windt, 1998) was successfully applied to model the phenomenon. However, the possible effects taking place in the PLE spectra were not addressed. The main purpose of the present work is to establish a new numerical technique and corresponding software to model the phenomenon of excitation radiation interference within thin films and to correct the shape of distorted PLE spectra.

\section{System requirements}

A freely available software module (wizard) named iCorrector (Buntov, 2012) has been developed by us to automate the correction procedure of thin film PLE spectra. The wizard has been created and compiled to MS Windows, executable by means of NI LabView 2011, and makes use of several optimization and signal-processing toolkits. Thus iCorrector requires the free run-time engine of LabView 2011 or newer (National Instruments, 2011) to be installed on Windows XP, Windows 7 or later systems.

The wizard may be installed by simple unpacking to the destination directory. In terms of visualization of the features in the graphical user interface, iCorrector is best viewed using monitors with SXGA resolution $(1280 \times 1024$ pixels $)$ or better; the minimum recommended resolution is XGA $(1024 \times 768$ pixels $)$. At the automatic optimization step the wizard takes about $60 \mathrm{Mb}$ of RAM and may occupy a modern dual-core CPU for several minutes.

In order to run iCorrector one must specify paths to at least three text files containing the experimental spectrum to be corrected and optical constants for both the film and substrate materials. The text files must be ASCII-encoded and tab-separated. The first column of the spectrum file should contain photon energies in $\mathrm{eV}$, and the second should contain the photoluminescence intensity. Optical constants files were taken from the IMD software distribution and contain three columns: wavelength in $\AA$, refraction factors and extinction factors. Optionally users may also input paths to an electric field distribution map (Windows bitmap) and an emission centres concentration profile. Examples of these files may be found in the iCorrector package.

\section{Numerical technique and corresponding wizard steps}

As a sample we consider a thin film placed on top of a semi-infinite substrate (see the sketch in Fig. 1). A light beam is incident from the vacuum ambient at an angle $\theta$ and is reflected from the film-substrate interface. The interference of the incident and reflected beams creates a standing wave having various intensities at different depths and light wavelengths. Such an effect modifies the observed PLE spectrum of the emission centres within the film. The iCorrector 


\section{computer programs}

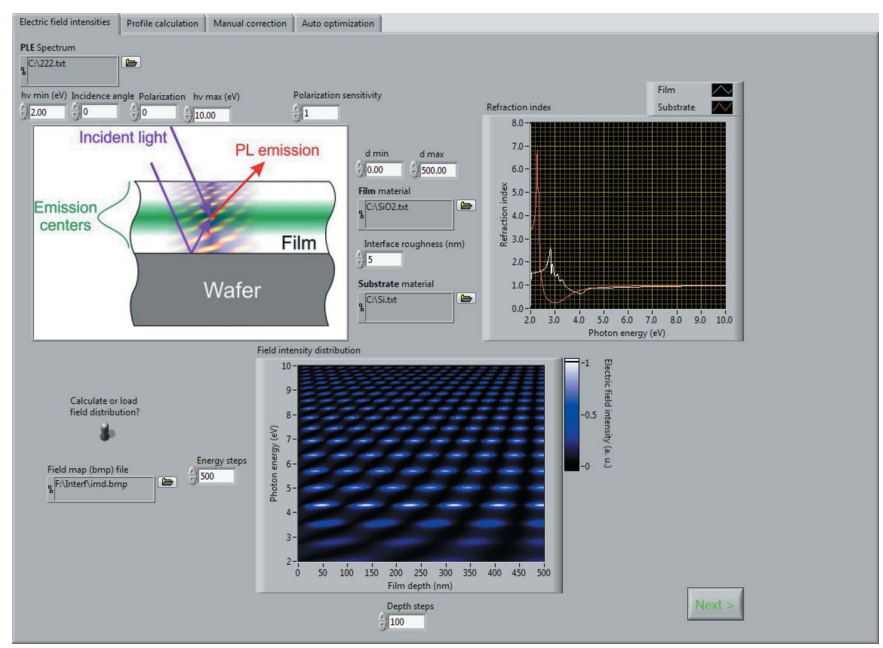

\section{Figure 1}

First page of the iCorrector wizard: modelling of the electric field intensity distribution within the film under study. An excitation radiation interference scheme for a thin transparent film placed on top of the reflecting wafer is depicted. The user specifies initial parameters (left) and material optical constants (right), and obtains a field intensity map (bottom).

wizard aims to restore the initial 'intrinsic' spectrum of photoluminescence (PL) centres. The overall correction process is divided into four major steps, which will be described in the following sections.

\subsection{Incident light intensities calculation}

The incident monochromatic light beam creates a standing wave within a thin transparent film on a substrate. In order to model the electric field intensity distribution throughout the film one can use an algorithm based on the recursive application of the Maxwell equations together with the Fresnel equations (Born \& Wolf, 1980), modified to include interfacial roughness and/or diffuseness (Stearns, 1989; Névot \& Croce, 1980). Described in detail by Windt (1998), this calculation scheme takes into account the incidence angle, film thickness and beam polarization factor $f$,

$$
f=\frac{I^{s}-I^{p}}{I^{s}+I^{p}}
$$

where $I^{s}$ and $I^{p}$ are the incident intensities for $s$ and $p$ polarizations, respectivley (perpendicular and parallel to the plane of incidence, respectively, so that the unpolarized radiation corresponds to $f=0$ ). All these parameters are specified on the first page of the iCorrector wizard (Fig. 1). The wavelength-dependent material refraction and extinction indexes for the film and the substrate are taken from tabdelimited text files. The software distribution contains optical constants for over 150 materials provided with the IMD software. However, a user can select any other material specifying an appropriate constants file. The calculations result in a two-dimensional map $I(z, h v)$ of the electric field intensity within the film showing its dependence on depth $z$ and incident photon energy $h v$.

The calculation of the electric field intensities was performed in our case for a $500 \mathrm{~nm} \mathrm{SiO}_{2}$ film on top of a semi-infinite silicon substrate. The excitation radiation with an incidence angle of $17^{\circ}$ and quanta energies of $4-10 \mathrm{eV}$ were assumed in full accordance with the experimental conditions of the SUPERLUMI station (Zatsepin et al., 2009). The obtained two-dimensional map of field intensity $I$ showing its dependence on film depth $z$ and excitation energy $h v$ is presented

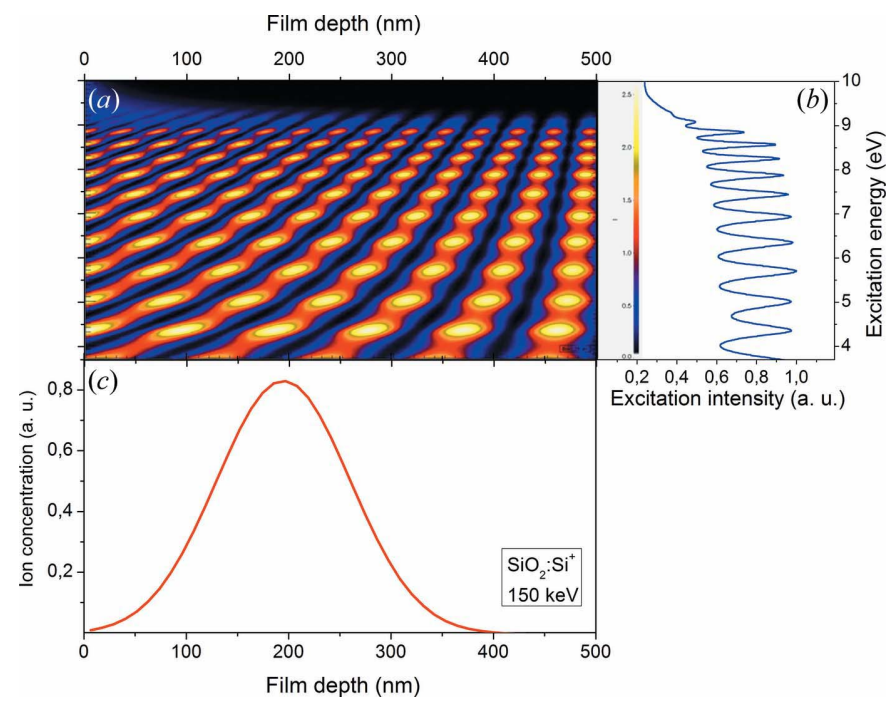

Figure 2

Excitation light interference within a thin ion-implanted $\mathrm{SiO}_{2}$ film on a silicon substrate. (a) Electric field intensity map showing its dependence on depth and photon energy. (b) Excitation radiation spectral profile with interference fringes. (c) Depth distribution of the concentration of the emission centres (implanted ions).

in Fig. 2(a). It reveals a set of interference fringes which may cause the PLE spectra oscillations.

\subsection{Interference profile modelling}

In order to model the interference pattern that distorts the PLE spectrum one should take into account the depth distribution of the luminescent centres. Such a profile might either be uniform or have a specific shape, which is typically described by the depth dependence read from a two-column text file. The centres concentration is often determined experimentally or simulated with a considerable error. In such a case iCorrector supports a smoothing procedure for the concentration profiles (Fig. 3).

In our case the emission centres are either point defects or nanoclusters formed by ion implantation. Both have a concentration

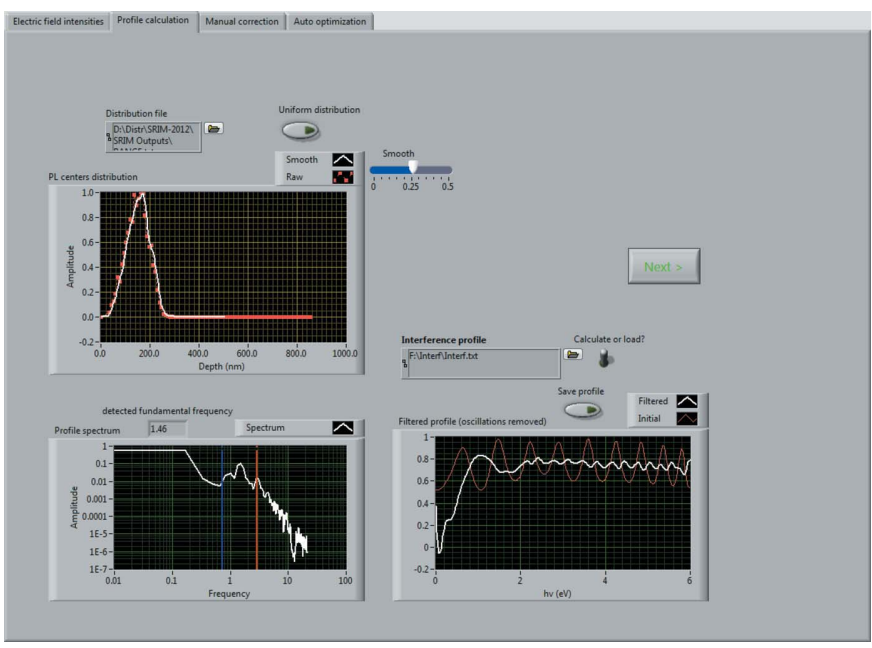

Figure 3

Second page of the iCorrector wizard: calculation of the interference profile affecting PLE spectra. The depth distribution of the luminescent centres (top graph) is taken into account. The band-stop filter frequencies are determined (bottom left) in order to eliminate interference fringes (bottom right). 
proportional to the initial ion implantation profile. The implanted ions distribution $C(z)$ was calculated by means of the SRIM-2012 software package (Ziegler \& Biersack, 2012). The depth profile for the $150 \mathrm{keV} \mathrm{Si}^{+}$ions is shown in Fig. 2(c).

In order to calculate the interference profile $I_{\text {int }}(h v)$, the electric field intensity map $I(z, h v)$ is integrated with respect to the depth $z$ using the ion distribution as a weighting function,

$$
I_{\text {int }}(h v)=\int_{0}^{d} I(z, h v) C(z) A(z) \mathrm{d} z,
$$

where $d$ is the film thickness, $C(z)$ is the ion implantation profile, and $A(z)$ is a coefficient accounting for the optical absorption of the luminescence radiation. The resulting interference pattern $I_{\text {int }}(h v)$, shown in Fig. 2(b), is proportional to the excitation light intensity (photons number) at the specific excitation energy and is valid for the specified film thickness, light incidence angle and distribution of the luminescent centres. It may be used regardless of the physical nature of the emission centres unless the concentration of the centres is high enough to alternate the optical properties of the host matrix. The latter case may be modelled by a specially arranged multilayer structure with a variable refraction index which is beyond the scope of the present article and is not considered here. The third term $A(z)$ is important when the thin film material has a considerable optical absorption at the emission wavelength. If the luminescence observed lies within the transparency gap of the host material, this term might be neglected.

\subsection{Manual correction of the PLE spectra}

An interference pattern may affect the intrinsic excitation spectrum (energy dependence of the photoluminescence quantum yield) $\eta_{\mathrm{PLE}}(h v)$, so that the instrument registers a distorted variant $\eta_{\mathrm{PLE}}^{*}(h v)$,

$$
\eta_{\mathrm{PLE}}^{*}(h v)=I_{\mathrm{int}}(h v) \eta_{\mathrm{PLE}}(h v) .
$$

Thus the main goal is to retrieve the undistorted initial spectrum using the model approximation of $I_{\text {int }}(h v)$. The most straightforward way to achieve the goal is to divide by $I_{\text {int }}(h v)$. However, the restoration of the experimental spectrum is actually complicated, for the following reasons:

(i) The roughness of the film interfaces affects the field intensity map and in most cases is not known exactly.

(ii) Although the film thickness is usually measured or calculated, it always contains an uncertainty.

(iii) The light incidence angle may be altered owing to inaccurate specimen positioning.

(iv) The SRIM-calculated ion distributions introduce an error of 4-6\% even if all parameters are specified properly.

(v) The equations used to model the electric field intensities are most of all phenomenological and also contribute to the overall error.

From Fig. 4 one may notice the way in which the most important parameters (film thickness, light incidence angle and distribution of the luminescent centres) influence the calculated interference profile. The thickness changes lead to scaling of the oscillations period: the thinner the film, the larger the distance between interference fringes (Fig. 4a). The growth of the incidence angle results in an energy shift of the oscillations (Fig. 4b). A narrow Gaussian distribution (12 nmwide peak at $60 \mathrm{~nm}$ depth) of the luminescent centres gives the highest excitation amplitude while a wide one (40 nm-wide maximum at $213 \mathrm{~nm}$ depth) gives the lowest, almost coinciding with the uniform distribution (Fig. 4c). In order to compensate for the uncertainties in

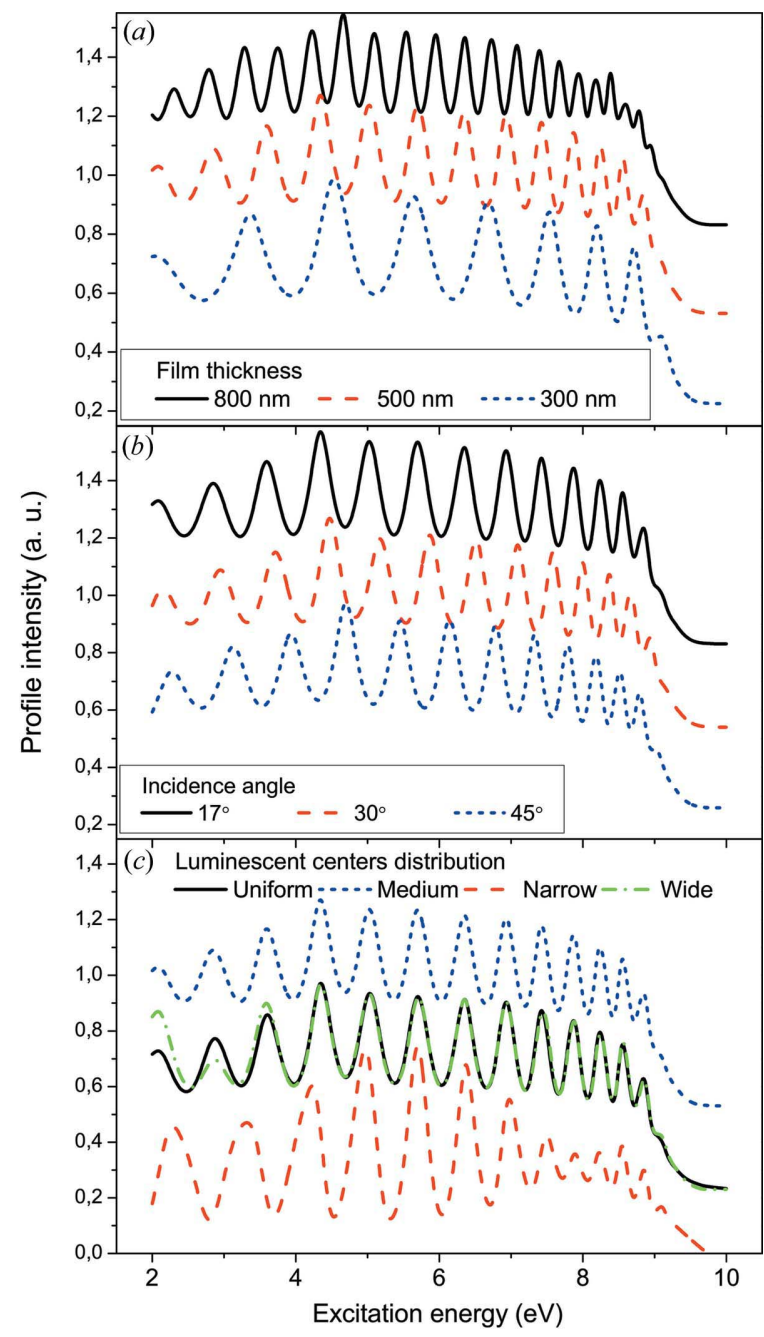

Figure 4

Interference profiles modelled for samples with various thicknesses $(a)$, excitation light incidence angles $(b)$ and luminescent centres distributions $(c)$, other parameters being equal. The curves presented were shifted along the intensity axis for convenience.

these parameters one must propose a more comprehensive method of correction. We suggest the following linear extension of (3), introducing four additional factors,

$$
\eta_{\mathrm{PLE}}(h v)=\frac{\eta_{\mathrm{PLE}}^{*}(h v)}{a_{2} I_{\mathrm{int}}\left(a_{1} h v+b_{1}\right)+b_{2}},
$$

where $a_{1}$ and $a_{2}$ are scaling factors for the energy and intensity, respectively; and $b_{1}$ and $b_{2}$ are shifts for the same quantities. The $b_{1}$ parameter is aimed to compensate for the incidence angle error, $a_{1}$ accounts for the film thickness uncertainty, and $a_{2}$ and $b_{2}$ may correct the accuracy of the distribution of the PL centres. The manual correction procedure is performed on the third step of the wizard. A user may select the spectral range in which the interference fringes are present by moving the blue and red cursors on the graph (Fig. 5). By changing the parameters $a_{1}, a_{2}, b_{1}$ and $b_{2}$ (named Profile energy scale, Intensity scale, Profile energy offset and Intensity offset, respectively) one can obtain the manually corrected spectrum (white line in Fig. 5) from the distorted one (red line) divided by a slightly scaled and shifted interference profile (green line). 


\section{computer programs}

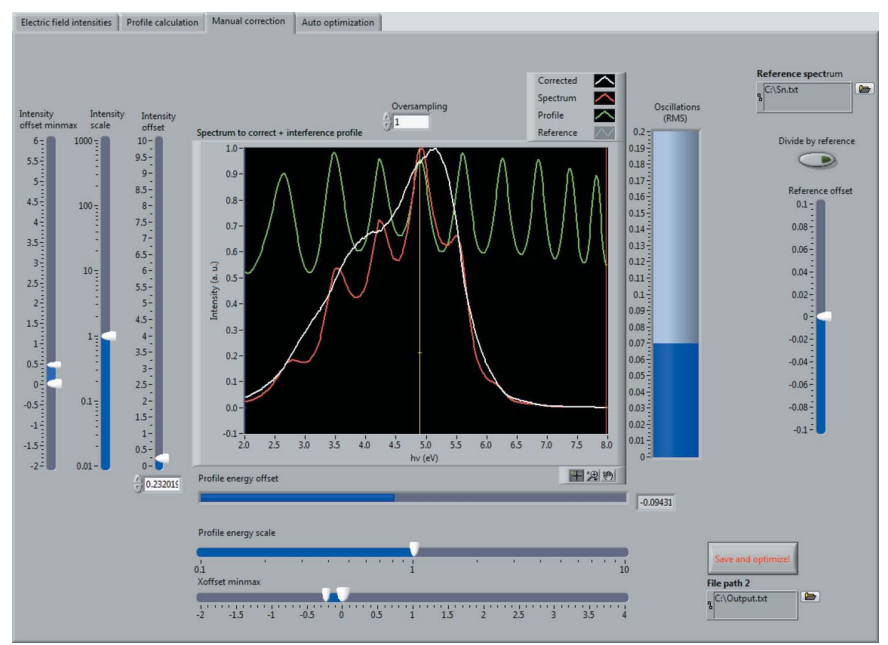

Figure 5

Third page of the iCorrector wizard: manual correction of the experimental spectrum. The user can select appropriate initial values for the fitting parameters by moving corresponding sliders and cursors.

\subsection{Automated optimization}

Numerical optimization on these parameters gives a better approximation of the initial undistorted PLE spectrum. The most essential criterion for such optimization is the absence or minimal amplitude of interference-related fringes and oscillations in the corrected spectrum. In order to distinguish and remove these oscillations we attempt to use frequency filtering, namely the third-order band-stop Butterworth filter (Smith, 1997) (Fig. 6a). The lower $\left(f_{1}\right)$ and higher $\left(f_{\mathrm{h}}\right)$ cut-off frequencies are taken from the spectrum of the $I_{\text {int }}(h v)$ interference profile (Fig. $\left.6 b\right)$ by multiplying its fundamental frequency $f_{\mathrm{f}}$ by factors 0.5 and 2 , respectively. These factor values give an adequate bandwidth for the filter, though they may be debated and changed manually in some particular cases. However, the values should be kept constant for all the spectra corrected with a single profile.

Having been filtered with the Butterworth filter the $I_{\text {int }}(h v)$ pattern lacks the majority of its oscillations and becomes smooth (see the black solid line in Fig. $6 a$ ). A similar effect is expected to be achieved for the PLE spectrum obtained from (3) when the optimized parameters values are used. The result of the evaluation of (3) is subjected to the corresponding band-pass filter with the same frequencies. The root-mean-square value of the filtered signal, containing mostly the interference-related oscillations, is then minimized in order to obtain the optimum parameters values. The iCorrector software allows the upper and lower boundaries to be specified for all the optimization parameters (Fig. 5) in order to avoid the unwanted or physically impossible value combinations.

Automatic optimization is performed at the fourth stage of the wizard. A non-linear optimization procedure attempts to select the best combination of fitting parameters within the specified boundaries in order to minimize the RMS value of the frequency-filtered spectrum (optimization criterion value). The criterion is calculated according to the following procedure. The experimental spectrum undergoes a Butterworth band-pass filter with the lower $f_{1}$ and higher $f_{\mathrm{h}}$ frequencies determined from the calculated interference profile. Then, in order to obtain a single measure of the oscillations level, the root-mean-square value is calculated from the filtered curve. The properly selected cut-off frequencies eliminate most of the unwanted oscillations, such as the vibronic series of lines. However, the user

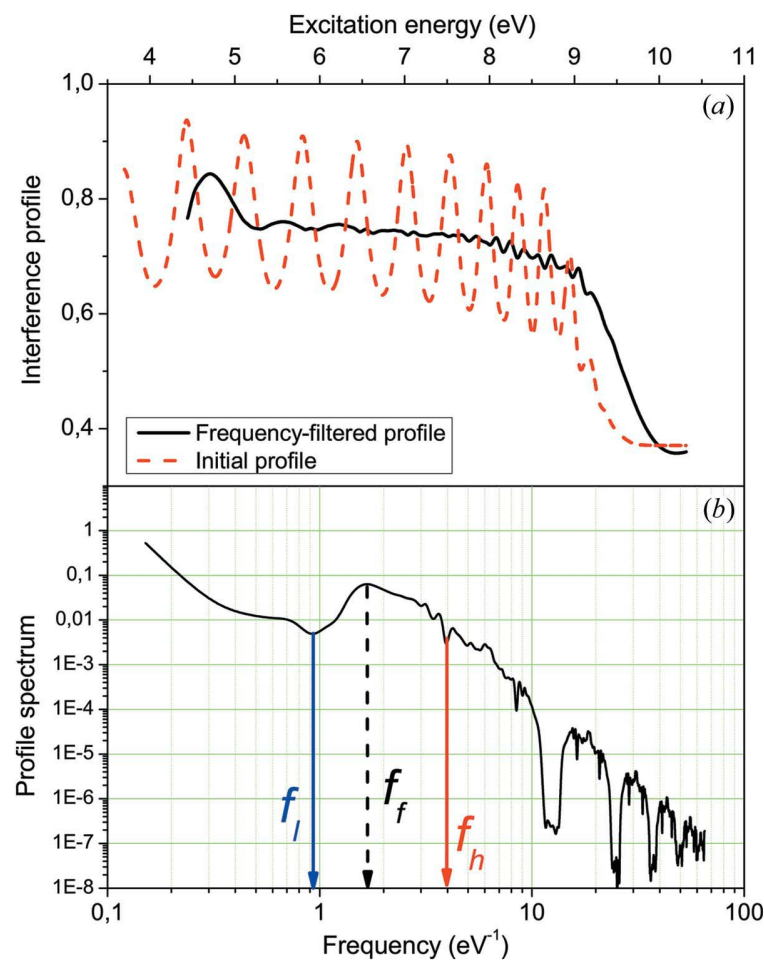

Figure 6

Example of the interference profile frequency filtering in order to eliminate the fringes. (a) Calculated profile before (dashed) and after (solid) third-order Butterworth filtering. (b) Frequency spectrum (Fourier transform) of the initial profile allows calculation of the filter's higher $f_{\mathrm{h}}$ and lower $f_{1}$ frequencies on the basis of the fundamental frequency $f_{\mathrm{f}}$.

should be careful and check whether the oscillations observed are caused by interference. For example, a change in the light incidence angle should considerably shift the interference fringes.

The last window of Fig. 7 presents the restored spectrum in comparison with the initial and manually corrected ones. The two intensity graphs show the optimization criterion and its dependence on the pair parameters $a_{1}-b_{1}$ and $a_{2}-b_{2}$. The red cross-like cursors can be moved to select these parameters and view the resulting spectrum on the central graph (red line on Fig. 7). The obtained results can be saved to a text file.

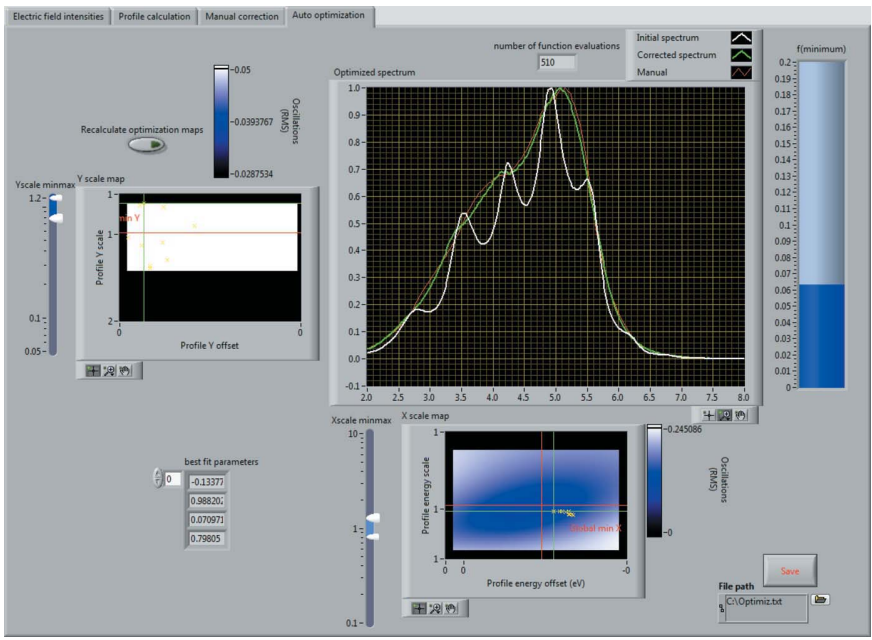

Figure 7

Final page of the iCorrector wizard: automated optimization results. A non-linear fitting procedure attempts to minimize the interference-related oscillations level. 


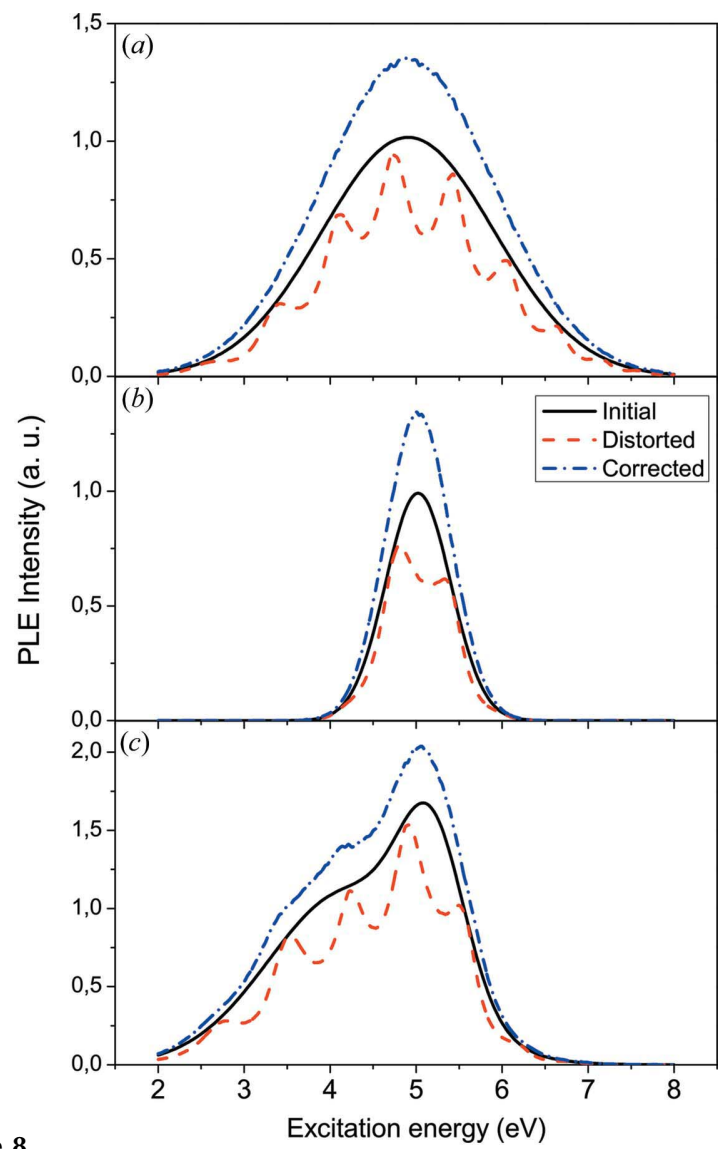

Figure 8

$$
\text { Excitation energy (eV) }
$$

Restoration of the simulated interference-distorted PLE spectra containing a wide $(a)$, narrow $(b)$ and a superposition of two Gaussian bands $(c)$.

\section{Examples of interference correction in PLE spectra}

\subsection{Model-based testing}

Fig. 8 shows the results of three model-based tests where the algorithm was applied to correct the Gaussians deliberately divided by the interference profile shown in Fig. $2(b)$. One can see from Figs. $8(a)$ and $8(b)$ that the technique proposed successfully restores the line shape for the peaks whose width is, respectively, four times and two times wider than the period of oscillations. Fig. 8(c) demonstrates an effective correction of the complex spectrum containing two wide bands. In the case of a narrow band or a maximum with a fine structure, having a width comparable with or less than the oscillations period, it is difficult to restore the shape automatically.

\subsection{Experimental PLE spectra restoration}

In order to probe the method proposed and to give some examples of spectral correction we will consider three systems containing different luminescence centres. One of the most pronounced interference effects has been observed for ion-implanted silica films on a silicon substrate (Zatsepin et al., 2009). The excitation spectrum of the $2.2 \mathrm{eV}$ PL band is shown by a black solid line in Fig. 9(a). One may notice that the high-energy part $(h v>7.5 \mathrm{eV})$ of the spectrum is not affected by oscillations while the low-energy part demonstrates almost equally spaced maxima similar to those found in the interference pattern [Figs. 2(a) and 2(b)]. The modelled profile does not predict such a sharp boundary at $7.5 \mathrm{eV}$. It can be suggested that the two parts of the PLE spectrum originate from different luminescent centres, some of which feel the interference effects and others do not.
Table 1

Spectroscopic parameters of the deconvoluted Gaussian components for the $\mathrm{SiO}_{2}: \mathrm{Si}^{+}$sample.

\begin{tabular}{llllllll}
\hline$E_{\max }(\mathrm{eV})$ & 4.45 & $\begin{array}{l}5.10 \\
(\mathrm{ODC})\end{array}$ & $\begin{array}{l}6.03 \\
\left(E_{\mathrm{S}}^{\prime}\right)\end{array}$ & 6.71 & $\begin{array}{l}7.09 \\
(\mathrm{ODC})\end{array}$ & $\begin{array}{l}7.68 \\
(\mathrm{ODC})\end{array}$ & $\begin{array}{l}8.67 \\
\text { (excitons) }\end{array}$ \\
\hline FWHM (eV) & 0.31 & 0.33 & 0.81 & 0.69 & 0.42 & 0.96 & 1.53 \\
Amplitude (a.u.) & 0.02 & 0.06 & 0.33 & 0.38 & 0.13 & 0.55 & 0.89 \\
\hline
\end{tabular}

Taking into account the spectral positions of the bands, the 7 $10 \mathrm{eV}$ region may be related to some silica excitons while the 4$7.5 \mathrm{eV}$ region is usually connected to the excitation of point defects. Extra evidence for their different nature is nanosecond- and microsecond-order decay kinetics observed for the low-energy and highenergy sides of the spectrum (Zatsepin et al., 2009). A direct application of the proposed algorithm to the PLE spectrum of $\mathrm{SiO}_{2}: \mathrm{Si}^{+}$ films leads to unwanted oscillations appearing in the high-energy region. In full conformance with the physical interpretation of the bands we have approximated the 7.5-10 eV wing of the spectrum by an auxiliary Gaussian function $\left[h v_{\max }=7.6 \mathrm{eV}\right.$, FWHM $=3 \mathrm{eV}$; dashed line in Fig. 9(a)]. Then this function was subtracted from the initial spectrum to obtain its interference-affected part [red (dashdotted line) line in Fig. 9(a)]. The latter part was corrected by the iCorrector module and then added to the $7.6 \mathrm{eV}$ band. The resulting spectrum is shown in Fig. 9(b). It still has a complex shape, which may be deconvoluted to six elementary components whose parameters are presented in Table 1 .

Thus the developed interference modelling and correction technique together with corresponding software allows an automated restoration of the initial PLE spectra demonstrating pronounced regular oscillations. Resulting spectra provide trustworthy and

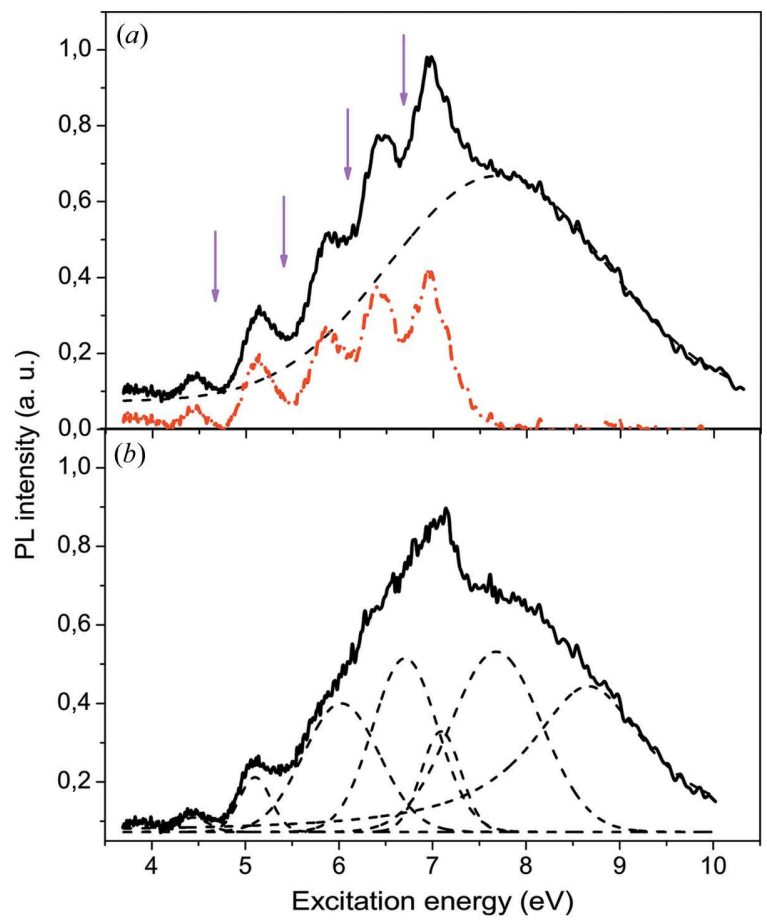

Figure 9

Correction of the PLE spectrum allows further spectroscopic analysis. (a) Experimental PLE spectrum (black solid line) of the $2.18 \mathrm{eV}$ PL band for the $\mathrm{SiO}_{2}: \mathrm{Si}^{+}$sample (Zatsepin et al., 2009) containing undistorted (dashed line) and distorted (dash-dotted line) parts. Arrows indicate the gaps introduced by interference fringes. $(b)$ The corrected spectrum lacks oscillations but keeps a complex shape. Deconvoluted Gaussian components are denoted by dashed lines. 
meaningful information about the point defects and excitons and can be further analysed by comprehensive spectroscopic methods including decomposition into elementary components. In particular, the spectroscopic features allow the $5.1 \mathrm{eV}, 7.09 \mathrm{eV}$ and $7.68 \mathrm{eV}$ PLE peaks (Fig. $8 b$ ) to be attributed to the singlet excited states of ODCtype defects (ODC - oxygen-deficient centres) (Skuja, 1998). The $6 \mathrm{eV}$ maximum is close to the optical absorption band of the $E_{\mathrm{S}}^{\prime}(\mathrm{Si}$ dangling bond at the interface) centre (Skuja, 1998). The band with the highest energy of $8.7 \mathrm{eV}$ has a Lorentzian shape and is connected to the $\mathrm{SiO}_{2}$ matrix exciton subsystem.

However, an interference pattern with less contrast may be observed in the excitation spectra when a different film thickness or a different luminescent centre is considered. In order to test the applicability of the correction techniques in the case of low-contrast fringes, different spectra of ion-implanted samples were studied. $\mathrm{SiO}_{2}: \mathrm{S}^{+}$films (Buntov et al., 2011) are known to contain molecular species $\left(\mathrm{S}_{2}\right.$ and $\mathrm{SO}_{2}$ molecules) which have visible PL bands along with several UV- and VUV-excitation maxima (Fig. 10a). On the other hand the $\mathrm{SiO}_{2}: \mathrm{Si}^{+}: \mathrm{C}^{+}$structures (Zatsepin et al., 2012b) have 1.5-2.8 eV photoluminescence introduced by $\mathrm{Si}, \mathrm{C}$ and $\mathrm{SiC}$ quantum dots, characterized by a complex PLE spectrum (Fig. 10a). Both samples have wide PLE spectra without well defined periodic oscillations. One may apply the correction procedure software even in this case. Restoration of the spectra has allowed the contribution of the interference fringes to be reduced significantly and false inflection points to be removed within the $5.5-8 \mathrm{eV}$ spectral range (Fig. 10b). The corrected PLE spectrum for sulfur-implanted samples resembles the known data for sulfur-doped silica fibres (Gerasimova et al., 2002), thus revealing the internal excited states of $\mathrm{S}_{2}$ and $\mathrm{SO}_{2}$ molecules. Thus, the presented examples show that the developed correction technique is valid for thin $\mathrm{SiO}_{2}$ films containing different types of luminescence centres: point defects, molecular species and quantum dots. We hope that the generality of the restoration algorithm will make it useful for other thin film systems as well.

\section{Conclusions}

In this study the role of interference effects during synchrotronradiation-excited photoluminescence experiments has been considered. It was shown that in the case of thin film and multilayer matrices a clear manifestation of the interference fringes within the UV and VUV range of photon energies complicates meaningful interpretation of PLE spectra. Therefore, such an effect requires a comprehensive method of spectral correction.

A new numerical technique was developed in order to address the problem of excitation radiation interference within thin films with an arbitrary distribution of PL emission centres. A corresponding algorithm aiming to correct the distorted PLE spectra was established. An objective correction criterion based on frequency-domain filtering of experimental spectra was suggested, ensuring the minimal amplitude of interference-related oscillations throughout the restored spectrum. The original iCorrector software program allowed automated restoration of the PLE spectra to be performed.

The software developed was successfully tested on both the simulated curves and experimentally acquired spectra of ionimplanted silicon dioxide films with quantum dots and point defects. The coincidence of the spectroscopic parameters of the deconvoluted components for the $\mathrm{SiO}_{2}: \mathrm{Si}^{+}$system with the known literature data for silica point defects and excitons confirms the correctness of

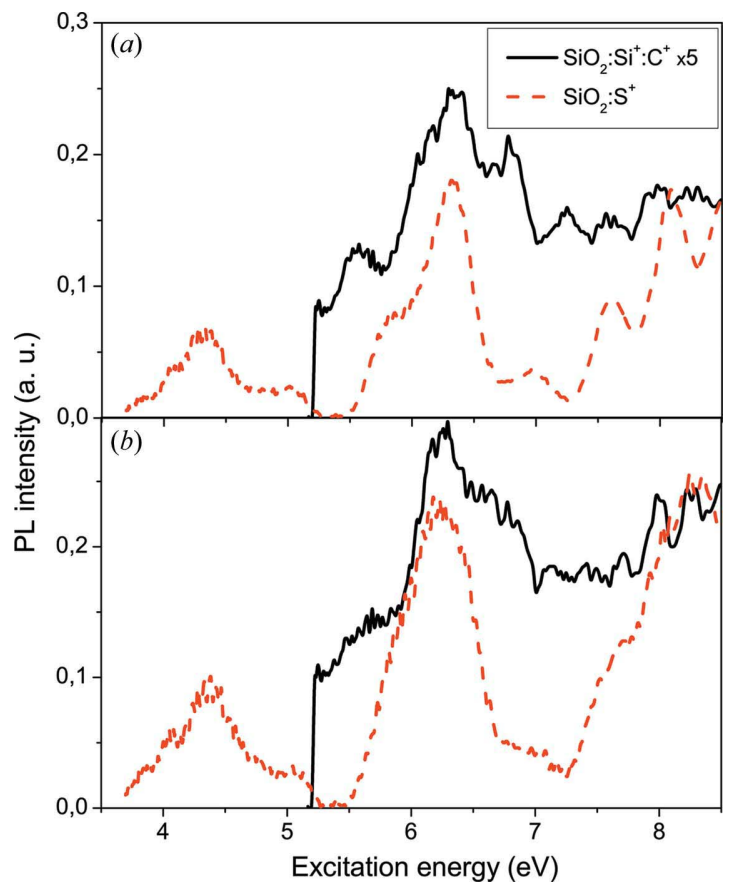

Figure 10

Correction procedure results for the $\mathrm{SiO}_{2}: \mathrm{Si}^{+}: \mathrm{C}^{+}$(Zatsepin et al., 2012b) and $\mathrm{SiO}_{2}: \mathrm{S}^{+}$(Buntov et al., 2011) samples. (a) Initial spectra. (b) Corrected spectra.

the restoration method. In examples of ion-implanted $\mathrm{SiO}_{2}: \mathrm{Si}^{+}$, $\mathrm{SiO}_{2}: \mathrm{Si}^{+}: \mathrm{C}^{+}$and $\mathrm{SiO}_{2}: \mathrm{S}^{+}$thin films the corrected spectra were shown to provide reliable spectral parameters of the luminescent centres.

The research was carried out in the framework of the RFBR project 13-08-00568 and was partially supported by Ural Federal University Development Program.

\section{References}

Born, M. \& Wolf, E. (1980). Principles of Optics, 6th ed. Oxford: Pergamon Press.

Buntov, E. A. (2012). iCorrector, http://sourceforge.net/projects/icorrector/.

Buntov, E., Zatsepin, A., Kortov, V., Pustovarov, V. \& Fitting, H. (2011). J. Non-Cryst. Solids, 357, 1977-1980.

Gerasimova, V. I., Rybaltovskii, A. O., Chernov, P. V. \& Zimmerer, G. (2002). Glass Phys. Chem. 28, 59-65.

Holm, R. T., McKnight, S. W., Palik, E. D. \& Lukosz, W. (1982). Appl. Opt. 21, 2512-2519.

National Instruments (2011). LabVIEW Run-Time Engine 2011, http:// joule.ni.com/nidu/cds/view/p/id/2534/.

Névot, L. \& Croce, P. (1980). Rev. Phys. Appl. 15, 761-779.

Skuja, L. (1998). J. Non-Cryst. Solids, 239, 16-48.

Smirani, R., Martin, F., Abel, G., Wang, Y., Chicoine, M. \& Ross, G. (2005). J. Lumin 115, 62-68.

Smith, S. W. (1997). The Scientist and Engineer's Guide to Digital Signal Processing. San Diego: California Technical Publishing.

Stearns, D. G. (1989). J. Appl. Phys. 65, 491-506.

Windt, D. L. (1998). Comput. Phys. 12, 360-370.

Zatsepin, A. F., Buntov, E. A., Kortov, V. S., Pustovarov, V. A., Fitting, H.-J., Schmidt, B. \& Gavrilov, N. V. (2012a). J. Surf. Invest. 6, 668-672.

Zatsepin, A. F., Buntov, E. A., Kortov, V. S., Tetelbaum, D. I., Mikhaylov, A. N. \& Belov, A. I. (2012b). J. Phys. Condens. Matter, 24, 045301.

Zatsepin, A., Fitting, H., Kortov, V., Pustovarov, V., Schmidt, B. \& Buntov, E. (2009). J. Non-Cryst. Solids, 355, 61-67.

Ziegler, J. F. \& Biersack, J. P. (2012). SRIM - The Stopping and Range of Ions in Solids, http://srim.org/\#SRIM. 